\title{
Generalization of Rician distribution for speckle pattern in ultrasound image processing and random flights problem in Shannon's signal space
}

\author{
Mitsuo Ohta, ${ }^{*}$ Shigeharu Miyata,* and Noboru Nakasako** \\ * Faculty of Engineering, Kinki University, \\ Umenobe 1, Takaya, Higashi-Hiroshima, 729-17 Japan \\ **Hiroshima Institute of Technology, \\ Miyake 2-1-1, Saeki-ku, Hiroshima, 731-51 Japan
}

(Received 26 August 1991)

\begin{abstract}
In the ultrasound diagnosis field, an analysis of speckle image texture in ultrasound Bscans is one of the important researches using stochastic signal processing method. In this paper, it is focused on finding out theoretically the probability density function of the speckle image texture for the magnitude of speckle image. First, the occurrence mechanism of speckle image texture is treated as a random walk problem in a multi-dimensional signal space based on Shannon's sampling theorem, by newly introducing a Hankel transformation type characteristic function. As a result, a unified probability density function expression has been explicitly proposed and it has been clarified that the present expression agrees with the well-known Rayleigh and Rician probability density functions as two special cases. Then, the legitimacy and the effectiveness of proposed stochastic analysis method has been experimentally confirmed by applying it to the speckle pattern of clinical B-scan image actually utilized for diagnostic purpose.
\end{abstract}

Keywords: Speckle image texture in ultrasound B-scans, Generalization of Rician distribution, Random flights problem, $N$-dimensional signal space, Characteristic function of Hankel transformation type

PACS number: 43. 80. Sh, 43. 35. Wa, 43. 40. Ng, 43. 35. Yb

\section{INTRODUCTION}

As is well-known, in the ultrasound diagnosis, ultrasonic measurements don't yet seem to be completely understood because of a fairly complicated physical factors, such as absorption, reflection, scattering of pulsed ultrasonic pressure waves from a tissue medium, and the electrically detecting apparatus for displaying backscattered or echo pulses. The image texture, or speckle, results from an interaction between the ultrasonic pulses transmitted into the body and the structures of the tissues. In general, there are two standpoints for analyzing the image texture. One is that the texture in the image of soft tissue is observed only as irregular or un- desirable noise. Several investigators on ultrasound B-scanning studied the character of ultrasound speckle only as random noise by using a stochastic analysis method. ${ }^{1-8)}$ On the other hand, another one is that the texture of the ultrasound B-scan image is latently associated with the microstructure of soft tissue as some regular signal and accordingly is fairly useful as a basis for diagnosis. ${ }^{9-13)}$

Our viewpoint is similar to the latter one since the reflection and the absorption of ultrasound wave are originally caused by the variation of acoustic impedance of a tissue medium, even if the correspondence between an image texture and a tissue texture greatly depends on the scatter's size and spatial distribution of the scatters within the pulse 
length of incident pulse. So, it seems to be possible to abstract some information on the objective structure if a skillful stochastic signal processing method of the image texture can be applied.

The stochastic researches (e.g., probability density expressions) on speckle pattern were discussed by many researchers in connection with the tissue characterization. Concretely, for the case of diffusedly scattering material with many fine particles, a Reyleigh probability density function for the intensity was already derived. ${ }^{1)}$ However, the statistical properties of ultrasound speckle need to be also applied to the general case of clinical image that tissue can be modeled as many of uniformly distributed small scatters. That is, in the case when the soft tissue has some microstructure, we have to try to find more unified probability density expressions for speckle due to the randomly distributed particles with a specular reflection. For this case, the probability density function for the intensity was expressed as a Rician probability density function. ${ }^{9)}$ Almost all of these previous studies treated only a typical case without a specular component, or were limited to a simple case when the signal is limited in a sufficiently narrow frequency band.

From the above point of view, this paper is focused on finding out theoretically how the probability. density function of the image texture for the intensity is affected by the frequency bandwidth $(: W)$ of the incident pulse, time constant $(: T)$ of the square law detector and the existence of a specular component $(: B)$. First, the occurrence mechanism of speckle image texture is treated as a random walk problem in a multi-dimensional signal space based on Shannon's sampling theorem, by newly introducing a Hankel transformation type characteristic function. As a result, a unified probability density function expression has been proposed explicitly and it has been clarified that the proposed unified expression agrees with the well-known Rayleigh and Rician probability density functions as two special cases. Then, the legitimacy and the effectiveness of proposed stochastic analysis method has been experimentally confirmed by applying it to the speckle pattern of clinical B-scan image actually utilized for diagnostic purpose.

\section{THEORETICAL CONSIDERATION}

$2.1 N$-Dimensional Random Walks Model for Generation of Ultrasound Image

In an ultrasound B-scan image, the pulsed incident ultrasound pressure wave $f(t)$ with a frequency bandwidth $W$ is observed as an intensity image texture resulting from an interaction between the structures of tissues and the coherent uitrasonic pulses transmitted into the body, after passing through the square law detector with an averaging time $T$. Of course, the above intensity of ultrasound consists of a signal component $S(t)$ from the regular tissue with specularity and a random noise component (i.e., speckle) $N(t)$ from the irregular tissue with diffusely scattering particles, after absorption and reflection caused by the regular and irregular variation of acoustic impedance. Of course, differing from the signal component, this random component $N(t)$ shows an undetermined property as a sum of arbitrary $s$ elemental echo components from an ensemble body of scattering particle with random phase and random amplitude caused by various type reflection and absorption from the irregular change of acoustic impedance based on the diffusedly scattering tissues (that is, it can be studied by functionally introducing an equivalent ensemble of $s$ scattering particles).

First, following Shannon's sampling theorem ${ }^{14)}$ on information theory, the above incident ultrasonic pressure wave $f(t)(=S(t)+N(t))$ is expressed as follows:

$$
f(t)=\sum_{n=0}^{2 T W} f\left(\frac{n}{2 W}\right) \frac{\sin (2 \pi W t-n \pi)}{2 \pi W t-n \pi}
$$

with

$$
f\left(\frac{n}{2 W}\right)=S\left(\frac{n}{2 W}\right)+N\left(\frac{n}{2 W}\right) \quad\left(N(t)=\sum_{i=1}^{s} N_{i}(t)\right) .
$$

Accordingly, the observed ultrasound intensity after passing through the square law detector in a B-scan image can be explicitly expressed as follows:

$$
\begin{aligned}
& \frac{1}{T} \int_{0}^{T} f^{2}(t) d t(=E) \\
& \quad=\sum_{n=0}^{2 T W}\left(\frac{1}{\sqrt{2 T W}}\left[S\left(\frac{n}{2 W}\right)+N\left(\frac{n}{2 W}\right)\right]\right)^{2} \\
& \quad=\sum_{n=0}^{2 T W} X_{n}^{2}
\end{aligned}
$$

with 


\section{OHTA et al.: ULTRASOUND IMAGE PROCESSING AND RANDOM FLIGHTS}

$$
\begin{aligned}
X_{n}= & \frac{1}{\sqrt{2 T W}}\left[S\left(\frac{n}{2 W}\right)+N_{1}\left(\frac{n}{2 W}\right)\right. \\
& \left.+N_{2}\left(\frac{n}{2 W}\right)+\cdots+N_{S}\left(\frac{n}{2 W}\right)\right] .
\end{aligned}
$$

On the other hand, in the problem of $N$-dimensional random walks, as shown in Fig. 1, from the fundamental relationship $\boldsymbol{r}_{j}=\sum_{k=1}^{N} \boldsymbol{e}_{k} x_{k j}$ (where $\boldsymbol{e}_{k}$ is a unit vector of $x_{k}$ axis), a composition of $s$ random vectors:

$$
\boldsymbol{R}=\sum_{j=1}^{s} \boldsymbol{r}_{j}
$$

can be reduced to a spherical sum in an $\mathrm{N}$-dimensional vector space as:

$$
R^{2}=\sum_{k=1}^{N} X_{k}^{2}\left(\text { with } X_{k}=\sum_{j=1}^{s} x_{k j}\right) .
$$

So, Eqs. (3) and (4) correspond to Eq. (6) with $N=$ $2 T W+1$ (for large number of $T$ and/or $W, N \cong$ $2 T W$ ). That is, since each coordinate component $X_{n}(n=1,2, \ldots, 2 T W+1)$ of a composition vector is given in Eq. (4) as a scalar sum of each coordinate component $S(n / 2 W)$ and $N_{i}(n / 2 W)(i=1,2, \ldots, s)$ for signal and noise ultrasound waves, the problem of evaluating the stochastic property of ultrasound intensity in a B-scan image can be mathematically studied as a stochastic problem on the vector composition $R$ in a Shannon's $N$-dimensional signal space $(N=2 T W+1 \cong 2 T W)$ by letting $R=\sqrt{E}$ (effective value) as shown in Fig. 2. Here, in Eqs. (1) and (2), the randomness of $f(t)$ is reflected in each $N_{i}(n / 2 W)$. After all, our present problem can be also recognized as a problem to derive the probability distribution of the composition $|\mathbb{R}|$ (or

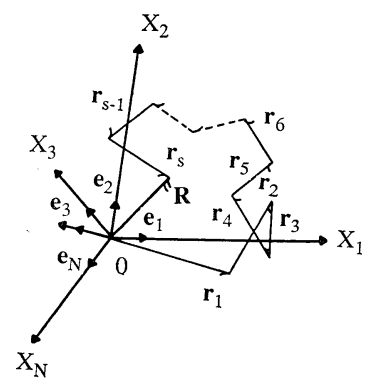

Fig. 1 Random walk in Shannon's $N$ dimensional signal space.

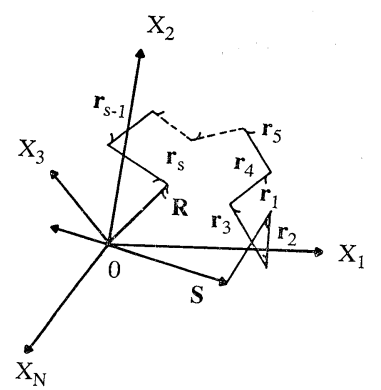

Fig. 2 Random walk accompanied by a regular component vector in Shannon's $N$-dimensional signal space.

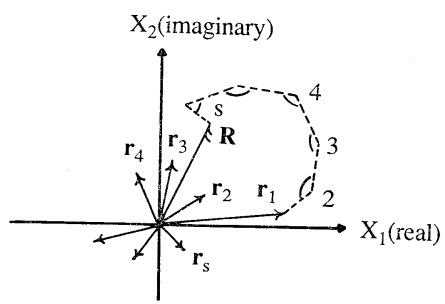

Fig. 3 Random walk in 2-dimensional (Gauss' complex) space.

$\left.E=R^{2}\right)$ by use of the statistical property of each $X_{k}$ (or $N_{i}(k / 2 W)$ ), in Shannon's multi-dimensional signal space (that is, by transformation $R=\sqrt{E}$ (effective value of $f(t)), X_{k}=(1 / \sqrt{2 T W}) f(K / 2 W)=$ $(1 / \sqrt{2 T W}) S(K / 2 W)+(1 / \sqrt{2 T W}) \sum_{i=1}^{s} N_{i}(K / 2 W)$ and $N=2 T W$, Eqs. (3) and (4) coincide with the relation of hypersphere sum in Eq. (6)).

Here, if the number $s$ of random flights tends to $\infty$, each of $X_{k}\left(=\sum_{j=1}^{s} x_{k j}\right)$ becomes to follow an independent Gaussian distribution through a central limit theorem. Furthermore, if the random ultrasonic pressure wave $N(t)$ is originally a white noise, the statistical property of randomness is reflected in each of $N(K / 2 W)$ (or $X_{k}$ ) and it follows an independent Gaussian distribution with mean 0 and constant variance. Here, in a special case with $N=$ 2 (see Fig. 3), it must be noticed that our present $N$-dimensional random flight problem in Fig. 1 just corresponds to the random signal composition problem of sinusoidal component waves in Gauss' complex plane. 
2.2 Introduction of Hankel Transform Type Characteristic Function in Multi-dimensional Signal Space

As is well-known, a joint probability density function $P\left(X_{1}, X_{2}, \ldots, X_{N}\right)$ can be given by an $N$-dimensional Fourier transformation of its joint characteristic function $F\left(\mu_{1}, \mu_{2}, \ldots, \mu_{N}\right)=\left\langle\exp \left\{i \sum_{k=1}^{N} \mu_{k} X_{k}\right\}\right\rangle$ as

$$
\begin{aligned}
P\left(X_{1}, X_{2}, \cdots, X_{N}\right) & \\
= & \left(\frac{1}{2 \pi}\right)^{N} \int_{-\infty}^{\infty} \cdots \int_{-\infty}^{\infty} \exp \left(-i \sum_{k=1}^{N} \mu_{k} X_{k}\right) \\
& \cdot F\left(\mu_{1}, \mu_{2}, \cdots, \mu_{N}\right) d \mu_{1} d \mu_{2} \cdots d \mu_{N} .
\end{aligned}
$$

Now, an $N$-dimensional polar coordinate $\left(\mu_{1}, \mu_{2}, \ldots\right.$, $\left.\mu_{N}\right) \rightarrow\left(\lambda, \phi_{1}, \ldots, \phi_{N-1}\right)$ with radius $\lambda$ of hypersphere as:

$$
\left\{\begin{array}{c}
\mu_{1}=\lambda \cos \phi_{1}, \\
\mu_{2}=\lambda \cos \phi_{2} \sin \phi_{1}, \\
\vdots \\
\mu_{k}=\lambda \cos \phi_{k} \prod_{j=1}^{k-1} \sin \phi_{j}(k=2,3, \cdots, N-1) \\
\vdots \\
\mu_{N}=\lambda \prod_{j=1}^{N-1} \sin \phi_{j} \\
\left(0 \leq \phi_{j} \leq \pi(j=1,2, \cdots, N-2), 0 \leq \phi_{N-1}<2 \pi\right)
\end{array}\right.
$$

is applied to Eq. (7). That is;

$$
\begin{aligned}
d \mu_{1} d \mu_{2} \cdots d \mu_{N} & =\lambda^{N-1} \prod_{j=1}^{N-1}\left(\sin \phi_{j}\right)^{N-j-1} d \phi_{j} d \lambda \\
& =d S_{(N)} \cdot d \lambda .
\end{aligned}
$$

Then, let us introduce a characteristic function in a form of the Hankel transform as the mean value of $F\left(\mu_{1}, \mu_{2}, \ldots, \mu_{N}\right)$ on the $N$-dimensional hypersphere surface as follows:

$$
\begin{aligned}
F(\lambda)= & \frac{1}{S_{(N)}} \int_{S_{(N)}} \cdots \\
& \cdot \int\left[F\left(\mu_{1}, \mu_{2}, \cdots, \mu_{N}\right)\right]_{\left(\mu_{1}, \mu_{2}, \cdots, \mu_{N}\right) \rightarrow\left(\lambda, \phi_{1}, \cdots, \phi_{N-1}\right)} \\
& \cdot d S_{(N)} \\
= & \frac{\Gamma((N / 2)+1)}{N(\sqrt{\pi})^{N}} \int_{0}^{2 \pi} \int_{0}^{\pi} \cdots \\
& \cdot \int_{0}^{\pi}\left[F\left(\mu_{1}, \mu_{2}, \cdots, \mu_{N}\right)\right]_{\left(\mu_{1}, \mu_{2}, \cdots, \mu_{N}\right) \rightarrow\left(\lambda, \phi_{1}, \cdots, \phi_{N-1}\right)} \\
& \cdot \prod_{j=1}^{N-1}\left(\sin \phi_{j}\right)^{N-j-1} d \phi_{1} d \phi_{2} \cdots d \phi_{N-1},
\end{aligned}
$$

where $d S_{(N)}$ is a surface element of $N$-dimensional hypersphere, $S_{(N)}$ is a total area of its surface and $S_{(N)}$ is a surface area $\lambda^{N-1}\left(N(\sqrt{\pi})^{N}\right) /(\Gamma(N / 2+1))$. Thus, from Eqs. (7) and (10), the following relation can be derived:

$P\left(X_{1}, X_{2}, \cdots, X_{N}\right) \propto \int_{0}^{\infty} \exp \left(-i \sum_{k=1}^{N} \mu_{k} X_{k}\right) F(\lambda) \lambda^{N-1} d \lambda$.

In terms of Eq. (6), the cumulative probability distribution $Q(R)\left(=\int_{0}^{R} P(R) d R\right)$ can be expressed by use of $P\left(X_{1}, X_{2}, \ldots, X_{N}\right)$ in Eq. (11) as follows:

$$
\begin{aligned}
Q(R)= & \iint_{X_{1}{ }^{2}+X_{2}{ }^{2}+\cdots+X_{N}{ }^{2} \leq R^{2}} \ldots \\
& \cdot \int P\left(X_{1}, X_{2}, \cdots, X_{N}\right) d X_{1} d X_{2} \cdots d X_{N} \\
\propto & \int_{0}^{\infty} G\left(\mu_{1}, \mu_{2}, \cdots, \mu_{N}\right) F(\lambda) \lambda^{N-1} d \lambda,
\end{aligned}
$$

where

$$
\begin{aligned}
& G\left(\mu_{1}, \mu_{2}, \cdots, \mu_{N}\right)=\iiint_{X_{1}{ }^{2}+X_{2}{ }^{2}+\cdots+X_{N}{ }^{2} \leq R^{2}} \cdots \\
& \cdot \int \exp \left(-i \sum_{k=1}^{N} \mu_{k} X_{k}\right) d X_{1} d X_{2} \cdots d X_{N} .
\end{aligned}
$$

Hereupon, the orthogonal transformation $\left((A)^{\mathrm{t}}(A)=\right.$ $(E))$ defined as

$$
\left(\begin{array}{c}
Y_{1} \\
Y_{2} \\
\vdots \\
\dot{Y}_{N}
\end{array}\right)=\left(\begin{array}{cccc}
a_{11} & a_{12} & \cdots & a_{1 N} \\
a_{21} & a_{22} & \cdots & a_{2 N} \\
\dot{0} & \vdots & \cdot & \cdot \\
\dot{a_{N 1}} & a_{N 2} & \cdots & a_{N N}
\end{array}\right)\left(\begin{array}{c}
X_{1} \\
X_{2} \\
\dot{5} \\
X_{N}
\end{array}\right)
$$

(abridged notation: $(Y)=(A)(X)$ )

with

$$
a_{1 k}=\mu_{k} / \lambda \quad \text { (see Eq. (8)) }
$$

is applied to Eq. (11) by considering the relation:

$$
\sum_{k=1}^{N} X_{k}^{2}=\sum_{k=1}^{N} Y_{k}^{2}
$$

and then one gets

$$
\begin{gathered}
G\left(\mu_{1}, \mu_{2}, \cdots, \mu_{N}\right)=\int \underset{Y_{1}{ }^{2}+Y_{2}{ }^{2}+\cdots+Y_{N} N^{2} \leq R^{2}}{\iint} \cdots \\
\cdot \int \exp \left(-i \lambda Y_{1}\right) d Y_{1} d Y_{2} \cdots d Y_{N}
\end{gathered}
$$$$
\text { with } \lambda^{2}=\sum_{K=1}^{N} \mu_{k}^{2} \text {. }
$$

Again, by using an $\mathrm{N}$-dimensional polar coordinate $(N \equiv 2 T W)$ : 


\section{OHTA et al.: ULTRASOUND IMAGE PROCESSING AND RANDOM FLIGHTS}

$$
\left\{\begin{array}{c}
Y_{1}=R \cos \theta_{1}, \\
Y_{2}=R \cos \theta_{2} \sin \theta_{1}, \\
\vdots \\
Y_{k}=R \cos \theta_{k} \prod_{j=1}^{k-1} \sin \theta_{j}(k=2,3, \cdots, N-1), \\
\vdots \\
Y_{N}=R \prod_{j=1}^{N-1} \sin \theta_{j} \\
\left(0 \leq \theta_{j} \leq \pi(j=1,2, \cdots, N-2), 0 \leq \theta_{N-1}<2 \pi\right), \\
d Y_{1} d Y_{2} \cdots d Y_{N}=R^{N-1} \prod_{j=1}^{N-1}\left(\sin \theta_{j}\right)^{N-j-1} d \theta_{j} d R,
\end{array}\right.
$$

Eq. (16) can be rewritten as follows:

$$
\begin{aligned}
G\left(\mu_{1}, \mu_{2}, \cdots, \mu_{N}\right) \\
\quad=\int_{0}^{R} \int_{0}^{\pi} e^{-i \lambda R \cos \theta_{1}}\left(\sin \theta_{j}\right)^{N-2} R^{N-1} d \theta_{1} d R \cdot C_{N-3} \\
\propto \int_{0}^{R} R^{N-1}(\lambda R)^{-\nu} J_{\nu}(\lambda R) d R \quad\left(\nu=\left(\frac{N}{2}-1\right)\right) .
\end{aligned}
$$

Here, the following integral formula has been used:

$$
J_{\nu}(z) \Gamma\left(\nu+\frac{1}{2}\right) \Gamma\left(\frac{1}{2}\right)=\left(\frac{z}{2}\right)^{\nu} \int_{0}^{\pi} e^{i z \cos \theta} \sin ^{2 \nu} \theta d \theta,
$$

where $C_{N-3}$ is defined by the definite integral:

$$
\begin{aligned}
C_{N-3}= & \int_{0}^{\pi}\left(\sin \theta_{2}\right)^{N-3} d \theta_{2} \int_{0}^{\pi}\left(\sin \theta_{3}\right)^{N-4} d \theta_{3} \cdots \\
& \cdot \int_{0}^{\pi}\left(\sin \theta_{N-2}\right) d \theta_{N-2} \int_{0}^{2 \pi} d \theta_{N-1} .
\end{aligned}
$$

By substituting Eq. (18) into Eq. (12), the probability density function of $R$ is expressed with use of a constant $K$ as:

$$
P(R)=K \int_{0}^{\infty} F(\lambda)(\lambda R)^{N / 2} J_{\nu}(\lambda R) d \lambda .
$$

Now, by newly introducing functions:

$$
\begin{aligned}
& F_{0}(\lambda)=\lambda^{m-(1 / 2)} F(\lambda), \\
& P_{0}(R)=\frac{1}{K} R^{-(m-(1 / 2))} P(R) \quad\left(m=\frac{N}{2}\right),
\end{aligned}
$$

the self dual relations of the Hankel transformation can be easily derived from Eq. (21) as:

$$
\left.\begin{array}{l}
P_{0}(R)=\int_{0}^{\infty} F_{0}(\lambda) J_{\nu}(\lambda R) \sqrt{\lambda R} d \lambda, \\
F_{0}(\lambda)=\int_{0}^{\infty} P_{0}(R) J_{\nu}(\lambda R) \sqrt{\lambda R} d R .
\end{array}\right\} \quad(\nu=m-1)
$$

In Eq. (23), it should be noted that $P_{0}(R)$ doesn't satisfy a normalized condition as a probability density function, differing from $P(R)$.

From Eq. (10), it can be guessed that $F(\lambda)$ has the following fundamental property as a characteristic function, differing from $F_{0}(\lambda)$ :

$$
\lim _{\lambda \rightarrow 0} F(\lambda)=1 \text {. }
$$

Here, the property $\lambda \rightarrow 0$ corresponds to $\mu_{1}=\mu_{2}=$ $\ldots=\mu_{N} \rightarrow 0$. On the other hand, by using the relation:

$$
\lim _{\lambda \rightarrow 0}(\lambda R)^{-\nu} J_{\nu}(\lambda R)=1 /\left\{2^{\nu} \Gamma(\nu+1)\right\},
$$

$F(\lambda)$ can be also expressed from Eqs. (22) and (23) as follows:

$$
\begin{aligned}
F(\lambda)= & \frac{1}{K} \int_{0}^{\infty}(\lambda R)^{-\nu} J_{\nu}(\lambda R) P(R) d R \underset{\lambda \rightarrow 0}{\rightarrow} 1 / \\
& \left\{K 2^{\nu} \Gamma(\nu+1)\right\} .
\end{aligned}
$$

From Eqs. (24) and (26), the constant $K$ is concretely determined as follows:

$$
K=1 /\left\{2^{m-1} \Gamma(m)\right\} .
$$

Accordingly, from Eqs. (21) and (26), the probability density function of the radius $R$ of hypersphere is explicitly obtained through the characteristic function method of Hankel transform type as follows:

$$
\begin{aligned}
P(R) & =\frac{R^{m}}{2^{m-1} \Gamma(m)} \int_{0}^{\infty} F(\lambda) \lambda^{m} J_{m-1}(\lambda R) d \lambda \\
F(\lambda) & =<2^{m-1} \Gamma(m) \frac{J_{m-1}(\lambda R)}{(\lambda R)^{m-1}}> \\
& =1+\sum_{n=1}^{\infty} \frac{(-1)^{n} \Gamma(m) \Omega_{n}}{2^{2 n} n ! \Gamma(m+n)} \lambda^{2 n}\left(\Omega_{n}=<R^{2 n}>\right) .
\end{aligned}
$$

For the self dual expression in Eq. (23), one can use $P_{0}(R)$ and $F_{0}(R)$ after substituting Eq. (27) into Eq. (22). From Eqs. (28) and (29), $P(R)$ is expressed in a form of moment statistics $\left\langle R^{2 n}\right\rangle$ with even order. When some of lower order moment statistics (e.g., $\Omega_{1}, \Omega_{2}$, etc.) are a priori known, the series expansion expression in Eq. (29) has the advantage of being able to derive the approximate expression of $P(R)$ from Eq. (28) after approximating $F(\lambda)$ as one compact function.

2.3 Probability Expression in the Form of Hankel Transform for an Effective Value of Ultrasonic Wave

If paying our attention not to the physical aspect 
that $R$ is based on the random flights problem as in Eqs. (5) and (6), but only to the resultant aspect that $R$ is a positive variable, another derivation process is available as shown below. Now, by applying the Weber-Schafheitlin's discontinuous integral of Bessel type:

$$
R^{\prime m} \int_{0}^{\infty} J_{m}\left(\lambda R^{\prime}\right) \frac{J_{m-1}(\lambda R)}{R^{m-1}} d \lambda= \begin{cases}1 & \left(R<R^{\prime}\right) \\ 0 & \left(R>R^{\prime}\right)\end{cases}
$$

to the discontinuous integral $D(R)$ as:

$$
\left.\begin{array}{c}
Q\left(R^{\prime}\right)=\int_{0}^{R^{\prime}} P(R) d R=\int_{0}^{\infty} P(R) D(R) d R \\
D(R)=1\left(R<R^{\prime}\right) \\
=0\left(R>R^{\prime}\right)
\end{array}\right\},
$$

the following expressions on cumulative probability function and probability density function are explicitly obtained:

$$
\begin{aligned}
& Q(R)=R^{m} \int_{0}^{\infty} J_{m}(\lambda R)<\frac{J_{m-1}(\lambda R)}{R^{m-1}}>d \lambda(m \geq 1 / 2), \\
& P(R)=\frac{d Q(R)}{d R}=R^{m} \int_{0}^{\infty} \lambda J_{m-1}(\lambda R)<\frac{J_{m-1}(\lambda R)}{R^{m-1}}>d \lambda .
\end{aligned}
$$

Though Eq. (33) superficially coincides only in its expression form with the result derived by substituting Eq. (29) into Eq. (28), it should be noteworthy that $F(\lambda)$ in Eq. (29) reflects originally the physical mechanism of Eq. (6) in a form of Eq. (10) and the statistical parameter $m$ is definitely determined as the half of dimension $N$.

2.4 General Integral Expression Type Probability Distribution for the Resultant Vector of $\mathrm{N}$ dimensional Random Flights

In the composition problem of $S$ random vectors $\left(\boldsymbol{R}=\left[X_{1}, X_{2}, \ldots, X_{N}\right], \boldsymbol{r}_{j}=\left[x_{1 j}, x_{2 n}, \ldots, x_{N j}\right]\right)$ as seen in Fig. 1 and in Eq. (5), based on the following statistical properties of each random flight given in advance, the general integral expression is explicitly derived for the probability $Q(R)$ or probability density $P(R)$ of the magnitude of a composite vector.

(a) each random walk $\boldsymbol{r}_{j}$ is independent of each other: This directly leads the following relation:

$$
\begin{aligned}
F\left(\mu_{1}, \mu_{2}, \cdots, \mu_{N}\right) & =<\exp \left(i \sum_{k=1}^{N} \sum_{j=1}^{s} \mu_{k} x_{k j}\right)> \\
& =\prod_{j=1}^{s}<\exp \left(i \sum_{k=1}^{N} \mu_{k} x_{k j}\right)>.
\end{aligned}
$$

From a form of exponential function, after applying the orthogonal transformation similar to that defined in Eq. (14):

$$
\left(\begin{array}{c}
y_{1 j} \\
y_{2 j} \\
\cdot \\
\cdot \\
y_{N j}
\end{array}\right)=\left(\begin{array}{cccc}
a_{11} & a_{12} & \cdots & a_{1 N} \\
a_{21} & a_{22} & \cdots & a_{2 N} \\
\cdot & \cdot & \cdot & \cdot \\
\cdot & \cdot & \cdot & \cdot \\
\cdot & \cdot & \cdot & \cdot \\
a_{N 1} a_{N 2} & \cdots & a_{N N}
\end{array}\right)\left(\begin{array}{c}
x_{1 j} \\
x_{2 j} \\
\cdot \\
\cdot \\
\cdot \\
x_{N j}
\end{array}\right) \quad\left(a_{1 k}=\mu_{k} / \lambda\right)
$$

to Eq. (34) with considering the relation $\sum_{K=1}^{N} y_{k j}{ }^{2}=$ $\sum_{K=1}^{N} x_{k j}{ }^{2}=r_{j}{ }^{2}$ and using an $N$-dimensional polar coordinate:

$$
\left\{\begin{array}{c}
y_{1 j}=r_{j} \cos \theta_{1 j}, \\
y_{2 j}=r_{j} \cos \theta_{2 j} \sin \theta_{1 j}, \\
: \\
\cdot \\
y_{k j}=r_{j} \cos \theta_{k j} \prod_{p=1}^{k-1} \sin \theta_{p j}(k=2,3, \cdots, N-1), \\
: \\
\cdot \\
y_{N j}=r_{j} \prod_{p=1}^{N-1} \sin \theta_{p j} \\
\left(0 \leq \theta_{p j} \leq \pi(p=1,2, \cdots, N-2), 0 \leq \theta_{N-1}<2 \pi\right),
\end{array}\right.
$$

one can get the following representation from the measure preserving law of probability:

$$
\begin{aligned}
& F\left(\mu_{1}, \mu_{2}, \cdots, \mu_{N}\right) \\
&=\prod_{j=1}^{s} \int_{-\infty}^{\infty} \int_{-\infty}^{\infty} \cdots \int_{-\infty}^{\infty} e^{i \lambda y_{1 j}} P\left(y_{1 j}, y_{2 j}, \cdots, y_{N j}\right) \\
& \quad \cdot d y_{1 j} d y_{2 j} \cdots d y_{N j} \\
&=\prod_{j=1}^{s} \int_{0}^{\infty} \int_{0}^{\pi} \cdots \int_{0}^{\pi} \int_{0}^{2 \pi} e^{i \lambda r_{j} \cos \theta_{1 j}} \\
& \quad \cdot P\left(r_{j}, \theta_{1 j}, \theta_{2 j}, \cdots, \theta_{N-1 j}\right) d r_{j} d \theta_{1 j} d \theta_{2 j} \cdots d \theta_{N-1 j} .
\end{aligned}
$$

(b) Magnitude and phase of random walks are independent of each other.

That is, the expectation operation in Eq. (37) can be separated into two ones with respect to phase and magnitude.

$$
\begin{aligned}
F\left(\mu_{1}, \mu_{2}, \cdots, \mu_{N}\right) \\
=\prod_{j=1}^{s} \int_{0}^{\infty}\left[\int_{0}^{\pi} \cdots \int_{0}^{\pi} \int_{0}^{2 \pi} e^{i \lambda r, \cos \theta_{1 j}}\right. \\
\left.\quad \cdot P\left(\theta_{1 j}, \theta_{2 j}, \cdots, \theta_{N-1 j}\right) d \theta_{1 j} d \theta_{2 j} \cdots d \theta_{N-1 j}\right] \\
\quad \cdot P\left(r_{j}\right) d r_{j} .
\end{aligned}
$$




\section{OHTA et al.: ULTRASOUND IMAGE PROCESSING AND RANDOM FLIGHTS}

(c) Expectation of each random flight with respect to phase is isotropic:

The isotropic expectation for phase of each elementary random flight $\boldsymbol{r}_{j}$ means that the probability density of $\boldsymbol{r}_{j}$ approaching arbitrary infinitesimal area element $d S_{(N) j}$ on the hypersphere with radius $\boldsymbol{r}_{j}$ (surface area $S_{(N) j}$ ) is constant $1 / S_{(N) j}$. That is:

$$
\begin{gathered}
P\left(\theta_{1 j}, \theta_{2 j}, \cdots, \theta_{N j}\right) d \theta_{1 j} d \theta_{2 j} \cdots d \theta_{N j}=\frac{1}{S_{(N) j}} d S_{(N) j}, \\
S_{(N) j}=r_{j}^{N-1} N(\sqrt{\pi})^{N} / \Gamma\left(\frac{N}{2}+1\right), \\
d S_{(N) j}=r_{j}^{N-1} \prod_{p=1}^{N-1}\left(\sin \theta_{p j}\right)^{N-p-1} d \theta_{p j} .
\end{gathered}
$$

After substituting Eq. (39) into Eq. (38) and using the integrated value $C_{N-3}$ in Eq. (20), the relation

$$
\begin{aligned}
F\left(\mu_{1}, \mu_{2}, \cdots, \mu_{N}\right)= & \sum_{j=1}^{s}\left\langle\frac{\Gamma((N / 2)+1)}{N(\sqrt{\pi})^{N}} C_{N-3}\right. \\
& \cdot \int_{0}^{\pi} e^{\left.i \lambda r_{j} \cos \theta_{1 j}\left(\sin \theta_{1 j}\right)^{N-2} d \theta_{1 j}\right\rangle}
\end{aligned}
$$

can be derived. Furthermore, by use of the integral formula in Eq. (19), one can obtain:

$$
\begin{aligned}
F\left(\mu_{1}, \mu_{2}, \cdots, \mu_{N}\right)= & \left(\Gamma\left(\frac{N}{2}\right)\right)^{s} \prod_{j=1}^{s} \\
& \cdot\left\langle\left(2 / \lambda r_{j}\right)^{(N / 2)-1} J_{(N / 2)-1}\left(\lambda r_{j}\right)\right\rangle,
\end{aligned}
$$

where $\langle>$ means the expectation operation with respect only to norm of each $\boldsymbol{r}_{j}$ and $\lambda=\sqrt{\sum_{K=1}^{N} \mu_{k}^{2}}$. By paying our attention to the fact that Eq. (41) is a function of only $\lambda$ and substituting it to Eq. (10), the probability density function of $N$-dimensional random walk $(N=2 T W)$ is explicitly derived through the relation in Eq. (28) as follows:

$$
\begin{aligned}
P(R)= & \left(\frac{1}{2}\right)^{N / 2-1}\left(\Gamma\left(\frac{N}{2}\right)\right)^{-1} R^{N / 2} \int_{0}^{\infty} F(\lambda) \lambda^{N / 2} \\
& \cdot J_{(N / 2)-1}(\lambda R) d \lambda \\
= & \left(\Gamma\left(\frac{N}{2}\right)\right)^{s-1}\left(\frac{1}{2}\right)^{N / 2-1} \int_{0}^{\infty}(\lambda R)^{N-2} \\
& \cdot J_{N / 2-1}(\lambda R) \prod_{j=1}^{s}\left\langle\frac{J_{(N / 2)-1}\left(\lambda r_{j}\right)}{\left(\lambda r_{j} / 2\right)^{N / 2-1}}\right\rangle d \lambda
\end{aligned}
$$

with

$$
F(\lambda)=\left(\Gamma\left(\frac{N}{2}\right)\right)^{s} \sum_{j=1}^{s}\left\langle\left(2 / \lambda r_{j}\right)^{N / 2-1} J_{N / 2-1}\left(\lambda r_{j}\right)\right\rangle .
$$

Furthermore, by using a well-known relation $(d / d u)\left(u^{\nu} J_{\nu}(u)\right)=u^{\nu} J_{\nu-1}(u)$, the following probability distribution function:

$$
\begin{aligned}
Q(R)= & R\left(\Gamma\left(\frac{N}{2}\right)\right)^{s-1} \int_{0}^{\infty}\left(\frac{1}{2} \lambda R\right)^{N / 2-1} \\
& \cdot J_{N / 2}(\lambda R) \prod_{j=1}^{s}\left\langle\frac{J_{N / 2-1}\left(\lambda r_{j}\right)}{\left(\lambda r_{j} / 2\right)^{N / 2-1}}\right\rangle d \lambda
\end{aligned}
$$

can be obtained. Here, only this expression form coincides apparently with Eq. (32) which doesn't reflect the physical mechanism of random walk.

2.5 Probability Density Expression for $N$-dimensional Random Flight Problem with No Regular Component

A. General theory (in a case with arbitrary number of $s$ )

Probability density function $P(R)$ for the $N$ dimensional random walks $r_{j}$ with arbitrary number of $s$ is considered in a special case when their random flights take the same constant magnitude: $\left|\boldsymbol{r}_{j}\right|=\boldsymbol{r}_{0}$. From Eq. (43), the characteristic function of the Hankel transformation type is first written as

$$
F(\lambda)=\left(\frac{\Gamma(m) 2^{m-1}}{\left(\lambda r_{0}\right)^{m-1}} J_{m-1}\left(\lambda r_{0}\right)\right)^{s} \quad(m=N / 2),
$$

which can be also rewritten as follows:

$$
\begin{gathered}
F(\lambda)=\exp (-s G(\lambda)), \\
\left.G(\lambda)=-\log \left\{\Gamma(m)\left(\frac{\lambda r_{0}}{2}\right)^{-(m-1)} J_{m-1}\left(\lambda r_{0}\right)\right\} \cdot\right\}
\end{gathered}
$$

Generally, by use of the well-known saddle point method, in an integral form:

$$
\int_{L} e^{-s f(\zeta)} u(\zeta) d \zeta \quad(R\{s f(\zeta)\}>0),
$$

what dominantly contributes to the integral $\int_{L}$ is only a domain near a minimal value of $R\{s f(\zeta)\}$, and this tendency becomes more obvious as $s$ becomes larger. That is, when substituting Eq. (46) into Eq. (42), owing to the saddle point method, what dominantly contributes to the integral of $P(R)$ is a zero point $\lambda=0$ (in the vicinity where $G(\lambda)$ itself becomes small) satisfying the minimal value of $G(\lambda)$ :

$$
G^{\prime}(\lambda)=r \cdot J_{m}(\lambda r) / J_{m-1}(\lambda r)=0 .
$$

When $s \rightarrow \infty$, it is sufficient to take into consideration only the vicinity of $\lambda=0$ for the integral form in Eq. (42). That is, by using the property in the vicinity of $\lambda=0$ : 


$$
\begin{aligned}
J_{\nu}(\lambda r) & =\left(\frac{\lambda r}{2}\right)^{\nu} \frac{1}{\Gamma(\nu+1)}\left\{1-\frac{\lambda^{2} r^{2}}{4(\nu+1)}+\cdots\right\} \\
& \cong\left(\frac{\lambda r}{2}\right)^{\nu} \frac{1}{\Gamma(\nu+1)} \exp \left\{-\frac{\lambda^{2} r^{2}}{4(\nu+1)}\right\},
\end{aligned}
$$

for the sufficiently large number of $s$, Eq. (45) becomes

$$
F(\lambda) \cong \exp \left\{-\frac{s r_{0}{ }^{2}}{4 m} \lambda^{2}\right\} \quad\left(\equiv F_{0}(\lambda), m=N / 2\right) .
$$

Thus, taking the well-known Levy's continuity theorem into consideration, let us reform Eq. (45) into a series expansion form as $F(\lambda)=F_{0}(\lambda)\{1+$ $\left.\sum_{n=1}^{\infty} Q_{n} \lambda^{2 n}\right\}$. At first, once after paying our attention to Eq. (50), $F(\lambda)$ in Eq. (45) is rewritten as follows:

$$
\left.\begin{array}{c}
F(\lambda)=\exp \left\{-\frac{s r_{0}{ }^{2}}{4 m} \lambda^{2}\right\} \cdot g(\lambda), \\
g(\lambda)=\exp \left\{+\frac{s r_{0}{ }^{2}}{4 m} \lambda^{2}\right\}\left\{\frac{\Gamma(m) 2^{m-1}}{\left(\lambda r_{0}\right)^{m-1}} J_{m-1}\left(\lambda r_{0}\right)\right\}^{s} \cdot
\end{array}\right\}
$$

By expanding each factor of $g(\lambda)$ in a form of the power series, a product of power series can be calculated on the basis of Cauchy's law and Abel's theorem:

$$
\begin{aligned}
g(\lambda)= & {\left[\sum_{k=0}^{\infty} \frac{\left(s r_{0}^{2}\right)^{k}}{k !(4 m)^{k}} \lambda^{2 k}\right]\left[\sum_{\imath=0}^{\infty} \sum_{\begin{array}{c}
t+u+v+\cdots=s \\
u+2 v+3 w+\cdots=\iota
\end{array}}\right.} \\
& \left.\cdot \frac{s !}{t ! u ! v ! w ! \cdots} D_{0}{ }^{t} D_{1}{ }^{u} D_{2}{ }^{v} \cdots\left(\lambda^{2} r_{0}{ }^{2}\right)^{u+2 v+3 w+\cdots}\right] \\
= & \sum_{n=0}^{\infty} Q_{n} \lambda^{2 n} \quad\left(Q_{0}=1\right)
\end{aligned}
$$

with

$$
D_{n}=\frac{(-1)^{n} \Gamma(m)}{2^{2 n} n ! \Gamma(m+n)}\left(D_{0}=1\right) .
$$

Here, the following relation is used:

$$
\begin{aligned}
& {\left[D_{0}+D_{1} X+D_{2} X^{2}+\cdots\right]^{s}} \\
& =\sum_{\imath=0}^{\infty} \sum_{\begin{array}{c}
t+u+v+\ldots=s \\
u+2 v+3 w+\cdots=\imath
\end{array}} \frac{s !}{t ! u ! v ! w ! \cdots} \\
& \text { - } D_{0}{ }^{t} D_{1}{ }^{u} D_{2}{ }^{v} \cdots X^{u+2 v+3 w+\cdots} \text {. }
\end{aligned}
$$

In Eq. (52), $Q_{n}$ can be calculated as

$$
\begin{aligned}
Q_{n}= & \sum_{\iota=0}^{n}\left[\sum_{\substack{t+u+v+\ldots=s \\
u+2 v+3 w+\cdots=\iota}} \frac{s !}{t ! u ! v ! w ! \cdots} D_{0}{ }^{t} D_{1}{ }^{u} D_{2}{ }^{v} \cdots\right] \\
& \cdot \frac{s^{n-1} r_{0}^{2 n}}{(n-1) !(4 m)^{n-\imath}},
\end{aligned}
$$

where $\sum_{\substack{t+u+v+\ldots=s \\ t+2 v+3 v+\cdots=t}}$ means a sum of different combinations of all values of positive integers $t, u, v, w, \ldots$ satisfying

$$
\left\{\begin{array}{l}
t+u+v+\cdots=s \\
t+2 v+3 v+\cdots=\iota .
\end{array}\right.
$$

For example, from Eq. (54), in order to calculate $Q_{1}$ and $Q_{2}$, it is enough to consider the following cases:

$$
\begin{aligned}
& n=1\left\{\begin{array}{l}
\iota=0: u=v=w=\cdots=0, t=s \\
\iota=1: u=1, v=w=\cdots=0, t=s-1,
\end{array}\right. \\
& n=2\left\{\begin{array}{l}
\iota=0: u=v=w=\cdots=0, t=s \\
\iota=1: u=1, v=w=\cdots=0, t=s-1
\end{array}\right. \\
& \iota=2\left\{\begin{array}{l}
u=2, v=w=\cdots=0, t=s-2 \\
u=0, v=1, w=\cdots=0, t=s-1 .
\end{array}\right.
\end{aligned}
$$

That is, one can get concretely

$$
\left.\begin{array}{c}
Q_{1}=\left(S D_{1}+\frac{s}{4 m}\right) r_{0}^{2}=0, \\
Q_{2}=\left({ }_{s} C_{2} D_{1}^{2}+S D_{2}+\frac{s^{2}}{4 m} D_{1}+\frac{s^{2}}{32 m^{2}}\right) r_{0}^{4} \\
=-s r_{0}^{4} / 32 m^{2}(m+1) .
\end{array}\right\}
$$

By substituting Eqs. (51), (52) and (56) into Eq. (42), the relation:

$$
\begin{aligned}
P(R)= & \frac{R^{m}}{2^{m-1} \Gamma(m)} \int_{0}^{\infty}\left[1+\sum_{n=2}^{\infty} Q_{n} \lambda^{2 n}\right] \\
& \cdot \exp \left\{-\frac{s r_{0}^{2}}{4 m} \lambda^{2}\right\} \lambda^{m} J_{m-1}(\lambda R) d \lambda
\end{aligned}
$$

is obtained. Here, by use of the integral formula on the Bessel function:

$$
\begin{gathered}
\int_{0}^{\infty} J_{\nu}(a t) t^{\mu-1} e^{-p^{2} t^{2}} d t=\frac{\Gamma((1 / 2)(\mu+\nu))(a / 2 p)^{\nu}}{\Gamma(\nu+1) 2 p^{\mu}} \\
\cdot \exp \left\{-\frac{a^{2}}{4 p^{2}}\right\}_{1} F_{1}\left(\frac{\nu-\mu}{2}+1 ; \nu+1 ; \frac{a^{2}}{4 p^{2}}\right)
\end{gathered}
$$

and the relation between the confluent hypergeometric function and the Laguerre polynomial:

$$
{ }_{1} F_{1}(-n ; m ; z)=\frac{n ! \Gamma(m)}{\Gamma(m+n)} L_{n}{ }^{(m-1)}(z),
$$

the following solution with respect to the probability density function can be derived:

$$
\begin{aligned}
P(R)= & \frac{2(N / 2)^{N / 2} R^{N-1}}{\Gamma(N / 2) s^{N / 2} r_{0}{ }^{N}} \exp \left\{-\frac{N R^{2}}{2 s r_{0}^{2}}\right\} \\
& \cdot\left\{1+\sum_{n=2}^{\infty} 2^{2 n} Q_{n} n ! \frac{(N / 2)^{n}}{s^{n} r_{0}^{2 n}} L_{n}^{(N / 2-1)}\left(\frac{N}{2 s r_{0}^{2}} R^{2}\right)\right\} .
\end{aligned}
$$

Here, the Laguerre polynomial defined as follows is used: 


\section{OHTA et al: : ULTRASOUND IMAGE PROCESSING AND RANDOM FLIGHTS}

$$
\begin{aligned}
L_{n}^{(\alpha)}(X) & =\frac{e^{X} X^{-\alpha}}{n !}\left(\frac{d}{d X}\right)^{n}\left\{e^{-X} X^{n+\alpha}\right\} \\
& =\sum_{j=0}^{n}\left(\begin{array}{c}
n+\alpha \\
n-j
\end{array}\right) \frac{(-X)^{j}}{j !} .
\end{aligned}
$$

B. Asymptotic expression (in a case with sufficiently large number of $s$ )

In a special case when the number $s$ of elementary random walks is sufficiently large, the following result is obtained in an asymptotic expression form:

$$
\begin{aligned}
P(R)= & \frac{2(N / 2)^{N / 2} R^{N-1}}{\Gamma(N / 2) s^{N / 2} r_{0}{ }^{N}} \exp \left\{-\frac{N R^{2}}{2 s r_{0}^{2}}\right\} \\
& \cdot\left\{1-\frac{2}{(N+2) s} L_{2}^{(N / 2-1)}\left(\frac{N}{2 s r_{0}^{2}} R^{2}\right)\right. \\
& \left.+O\left(1 / s^{2}\right) L_{3}^{(N / 2-1)}\left(\frac{N}{2 s r_{0}^{2}} R^{2}\right)\right\} .
\end{aligned}
$$

From the above equation, for 2- and 3-dimensional random walk problems, the following asymptotic expressions can be respectively derived in more explicit form by using Eq. (61):

(a) $N=2$,

$$
\begin{aligned}
P(R)= & \frac{2 R}{s r_{0}^{2}} \exp \left\{-\frac{R^{2}}{s r_{0}^{2}}\right\} \\
& \cdot\left\{1-\frac{1}{4 s}\left(2-\frac{4 R^{2}}{s r_{0}^{2}}+\frac{R^{4}}{s^{2} r_{0}^{4}}\right)+O\left(1 / s^{2}\right)\right\},
\end{aligned}
$$

(b) $N=3$,

$$
\begin{aligned}
P(R)= & \frac{3 \sqrt{6}}{\sqrt{\pi}} R^{2} \frac{1}{s^{3 / 2} r_{0}^{3}} \exp \left\{-\frac{3 R^{2}}{2 s r_{0}^{2}}\right\} \\
& \cdot\left\{1-\frac{3}{20 s}\left(5-\frac{10 R^{2}}{s r_{0}{ }^{2}}+\frac{3 R^{4}}{s^{2} r_{0}^{4}}\right)+O\left(1 / s^{2}\right)\right\} .
\end{aligned}
$$

Equation (63) is also a solution for the problem of random phase in Gauss' complex plane. By paying our attention to the following relation derived directly from Eq. (5):

$$
\begin{aligned}
\Omega_{1}= & \left\langle R^{2}\right\rangle=\langle\boldsymbol{R} \cdot \boldsymbol{R}\rangle=\left\langle\left(\sum_{i}^{s} \boldsymbol{r}_{i}\right)\left(\sum_{j}^{s} \boldsymbol{r}_{i}\right)\right\rangle \\
= & \sum_{i}^{s} \sum_{j}^{s}\left\langle\boldsymbol{r}_{i} \cdot \boldsymbol{r}_{i}\right\rangle=\sum_{j}^{s} \boldsymbol{r}_{0}{ }^{2} \\
= & s \cdot \boldsymbol{r}_{0}{ }^{2} \text { (in a case with the same constant } \\
& \text { magnitude: } \left.\left\langle\boldsymbol{r}_{i} \cdot \boldsymbol{r}_{j}\right\rangle=\delta_{i j} \boldsymbol{r}_{0}{ }^{2}\right),
\end{aligned}
$$

the first expansion term of Eq. (63) coincides with Rayleigh distribution form. The above Eqs. (63) and (64) are exactly the same as two asymptotic expressions with no use of Laguerre polynomial derived from a different viewpoint by $\mathrm{L}$. Rayleigh. Since Eq. (60) agrees with the previous well-known results in two special cases with $N=2$ and 3, the validity of Eq. (60) for the general type random flight problem in an $\mathrm{N}$-dimensional space can be partly guessed.

Furthermore, as guessed from the saddle point method for $s \rightarrow \infty$, the expression of $P(R)$ derived by substituting Eq. (50) into Eq. (42) becomes just the first term of series expansion type solution in Eq. (60) by use of Eq. (58), which is also obvious directly form Eq. (60) with $s \rightarrow \infty$.

From a superficial viewpoint only in the expression form, Eq. (60) must be formally included in a special case with $X=R^{2}$ and $S=\Omega_{1} / m$ in the following statistical Laguerre orthogonal expansion which was reported previously by paying our attention only to the positive fluctuation domain of random variable $X$ :

$$
\begin{aligned}
P(X)= & \frac{1}{\Gamma(m) S^{m}} X^{m-1} e^{-X / S}\left\{1+\sum_{n=2}^{\infty} \frac{n ! \Gamma(m)}{\Gamma(n+m)}\right. \\
& \left.\cdot\left\langle L_{n}{ }^{(m-1)}\left(\frac{X}{S}\right)\right\rangle L_{n}{ }^{(m-1)}\left(\frac{X}{S}\right)\right\} .
\end{aligned}
$$

That is, by use of probabilistic measure preserving property based on the transformation $X=R^{2}$, one directly has

$$
\begin{aligned}
P(R)= & \frac{2 m^{m} R^{2 m-1}}{\Gamma(m) \Omega_{1}{ }^{m}} \exp \left\{-\frac{m R^{2}}{\Omega_{1}}\right\} \\
& \cdot\left\{1+\sum_{n=2}^{\infty} 2^{2 n} C_{n} n !\left(\frac{m}{\Omega_{1}}\right)^{n} L_{n}{ }^{(m-1)}\left(\frac{m}{\Omega_{1}} R^{2}\right)\right\} .
\end{aligned}
$$

Here, by use of Eq. (61), $C_{n}$ is expressed as follows:

$$
\begin{aligned}
& C_{n}= \frac{n ! \Gamma(m)}{\Gamma(n+m)}\left\langle L_{n}^{(m-1)}\left(\frac{R^{2}}{\left(\Omega_{1} / m\right)}\right)\right\rangle \\
&=\sum_{K=0}^{n} \frac{(-1)^{K} \Gamma(m) \Omega_{K}}{2^{2 K} K ! \Gamma(m+K)} \cdot \frac{\Omega_{1}{ }^{n-K}}{(n-K) !(4 m)^{n-K}} \\
& \quad\left(\Omega_{k}=\left\langle R^{2 K}\right\rangle\right) .
\end{aligned}
$$

Equation (67) coincides with the result using the product of series based on Cauchy's law after taking a general series expression Eq. (29) in a place of Eq. (45) composing the second factor in Eq. (51). However, in order to give the statistical reflection into Eqs. (67) and (68) as a specific random walk problem as in Eqs. (54) and (60), it is necessary to make a calculation similar to Eq. (65) and the same kind of calculation on each of $\Omega_{k}=\left\langle R^{2 k}\right\rangle$ by use of Eq. (5). It is fairly troublesome. But, in a simplified case with a weak correlation among random walks, the latter may be easier in a method of analysis. It is somewhat easier to derive an approxi- 
mate expression like Eq. (62) instead of an exact expression in Eq. (60) even from Eqs. (67) and (68). In the previous report, ${ }^{15)}$ as the statistical parameter $m$, the estimate $\langle X\rangle^{2} / \sigma_{X}{ }^{2}$ was adopted by directly applying the moment method to the experimentally sampled data. In this paper, it is noteworthy that $\sum_{n=2}^{\infty}$ must be used instead of $\sum_{n=3}^{\infty}$ in Eq. (67) because $m$ is fixed as $N / 2$ directly connected with the dimension of signal space in advance.

2.6 Probability Density Expression for $N$-dimensional Random Flight Problem with a Regular Component for a Limiting Case $s \rightarrow \infty$

In general, a regular (or signal) vector $S$ can be mathematically treated in the same stochastic style as irregular (or random) vectors $N_{i}(i=1,2, \ldots, s)$ in Fig. 2, following to the well-known suggestion in the statistical communication theory. Accordingly, the characteristic function of Hankel transformation type for the present case in directly derived in the similar way as Eq. (45):

$$
F(\lambda)=\frac{\Gamma(m) 2^{m-1}}{(\lambda A)^{m-1}} J_{m-1}(\lambda A)\left[\frac{\Gamma(m) 2^{m-1}}{\left(\lambda r_{0}\right)^{m-1}} J_{m-1}\left(\lambda r_{0}\right)\right]^{s}
$$

with

$$
m=N / 2, A=|S| \text { and } r_{0}=\left|N_{i}\right| \text { for } i=1,2, \cdots, s .
$$

Especially, for the limiting case with sufficiently large number of $s$, by using the same saddle point method as in section 2.5, Eq. (69) can be asymptotically expressed as:

$$
F(\lambda)=\exp \left\{-\frac{\Omega_{1}}{4 m} \lambda^{2}\right\} \frac{\Gamma(m) 2^{m-1}}{(\lambda A)^{m-1}} J_{m-1}(\lambda A) \quad(m=N / 2)
$$

with $\Omega_{1}\left(\equiv\langle E\rangle=\left\langle R^{2}\right\rangle\right)=s r_{0}^{2}$. By substituting Eq. (70) into Eq. (42), the objective probability density function of an effective value $R$ can be derived as follows:

$$
\begin{aligned}
P(R)= & \frac{2 m R^{m}}{\Omega_{1}-A^{2}}\left(\frac{1}{A}\right)^{m-1} \\
& \cdot \exp \left\{-\frac{m\left(R^{2}+A^{2}\right)}{\Omega_{1}-A^{2}}\right\} I_{m-1}\left(\frac{2 m A R}{\Omega_{1}-A^{2}}\right), \\
P(E)= & \frac{m}{m_{T}-E_{0}}\left(\sqrt{\frac{E}{E_{0}}}\right)^{m-1} \\
& \cdot \exp \left\{-\frac{m\left(E+E_{0}\right)}{m_{T}-E_{0}}\right\} I_{m-1}\left(\frac{2 m \sqrt{E E_{0}}}{m_{T}-E_{0}}\right)
\end{aligned}
$$

with $m_{T} \equiv\langle E\rangle=\left\langle R^{2}\right\rangle$ and $E_{0} \equiv A^{2}$.

Here, the following integral relation ${ }^{16)}$ has been employed:

$$
\begin{aligned}
& \int_{0}^{\infty} e^{-p^{2} t^{2}} J_{\nu}(a t) J_{\nu}(b t) t d t \\
& =\frac{1}{2 p^{2}} \exp \left(-\frac{a^{2}+b^{2}}{4 p^{2}}\right) I_{\nu}\left(\frac{a b}{2 p^{2}}\right) \\
& \quad(\operatorname{Re}(\nu)>-1,|\operatorname{Arg}(p)|<\pi / 4) .
\end{aligned}
$$

By defining the non-dimensional variables:

$$
v=R / \sigma_{\mathrm{R}}, \quad a=A / \sigma_{\mathrm{R}} \quad\left(\sigma_{\mathrm{R}}{ }^{2}=\left(\Omega_{1}-A^{2}\right) / 2 m\right),
$$

Eq. (71) can be rewritten as

$$
P(v)=v^{m} a^{1-m} \exp \left\{-\frac{1}{2}\left(v^{2}+a^{2}\right)\right\} I_{m-1}(a v) .
$$

Furthermore, by using Eqs. (71) and (75), the moment statistics of the $K$ th order can be derived as follows:

$$
\begin{aligned}
\overline{R^{k}}= & \left(\frac{\Omega_{1}-A^{2}}{m}\right)^{k / 2} \frac{\Gamma(m+k / 2)}{\Gamma(m)} \\
& \cdot{ }_{1} F_{1}\left(-\frac{k}{2} ; m ;-\frac{m A^{2}}{\Omega_{1}-A^{2}}\right), \\
\overline{v^{k}}= & 2^{k / 2} \exp \left(-\frac{a^{2}}{2}\right) \frac{\Gamma(m+k / 2)}{\Gamma(m)} \\
& \cdot{ }_{1} F_{1}\left(m+\frac{k}{2} ; m ; \frac{a^{2}}{2}\right),
\end{aligned}
$$

after employing the power series expression of Bessel function and the Kummer's first transformation of confluent hypergeometric series:

$$
\begin{aligned}
& I_{\nu}(z)=\sum_{n=0}^{\infty} \frac{(z / 2)^{\nu+2 n}}{n ! \Gamma(\nu+n+1)}, \\
& e^{Z} \cdot F(\gamma-\alpha ; \gamma ;-z)=F(\alpha ; \gamma ; z) .
\end{aligned}
$$

Also, the cumulative distribution functions which are very important in the actual engineering field can be obtained as follows:

$$
\begin{aligned}
Q(R)= & \exp \left\{-\frac{m\left(R^{2}+A^{2}\right)}{\Omega_{1}-A^{2}}\right\} \sum_{n=0}^{\infty}\left(\frac{R}{A}\right)^{m+n} \\
& \cdot I_{m+n}\left(\frac{2 m A R}{\Omega_{1}-A^{2}}\right), \\
Q(v)= & \exp \left(-\frac{v^{2}+a^{2}}{2}\right) \sum_{n=0}^{\infty}\left(\frac{v}{a}\right)^{m+n} I_{m+n}(a v),
\end{aligned}
$$

corresponding to the probability density expression of Eqs. (71) and (75), after using the following relation:

$$
z^{\nu-k} I_{\nu-k}(z)=\left(\frac{d}{z d z}\right)^{k}\left\{z^{\nu} I_{\nu}(z)\right\} .
$$

Accordingly, from Eq. (80), the probability $Q(A)$ which is important as one of threshold signal levels is directly given as follows: 


\section{OHTA et al: : ULTRASOUND IMAGE PROCESSING AND RANDOM FLIGHTS}

$$
\begin{aligned}
Q(A)= & \frac{1}{2}\left[1-\exp \left\{-\frac{2 m A^{2}}{\Omega_{1}-A^{2}}\right\}\right. \\
& \left.\cdot \sum_{\nu=-(m-1)}^{(m-1)} I_{\nu}\left(\frac{2 m A^{2}}{\Omega_{1}-A^{2}}\right)\right] .
\end{aligned}
$$

This expression can also be derived from Eq. (71) by using the following integral relation:

$$
\begin{aligned}
& \int_{0}^{c} u^{n+1} \exp \left(-u^{2}\right) I_{n}(2 c u) d u \\
& \quad=\frac{1}{4} c^{n} \exp \left(c^{2}\right)\left[1-\exp \left(-2 c^{2}\right) \sum_{\nu=-n}^{n} I_{\nu}\left(2 c^{2}\right)\right] .
\end{aligned}
$$

[1] When a regular component $A$ is extremely larger than random components, Eq. (71) becomes a Gaussian type probability density distribution:

$$
P(R) \cong N\left(R ; A, \sigma_{R}^{2}\right)=\frac{1}{\sqrt{2 \pi} \sigma_{R}} \exp \left\{-\frac{(R-A)^{2}}{2 \sigma_{R}{ }^{2}}\right\},
$$

by using the following asymptotic property for large values of $z$ :

$$
I_{\nu}(z) \cong \frac{1}{\sqrt{2 \pi z}} e^{z} .
$$

After employing the probabilistic measure preserving transformation and the non-dimensional quantities:

$$
\begin{aligned}
& X=\frac{S m}{\Omega_{1}-A^{2}} R^{2}, \\
& B=\frac{S m}{\Omega_{1}-A^{2}} A^{2},
\end{aligned}
$$

the following unified expression of probability density function can be directly derived from Eqs. (71) and (72) as:

$$
\begin{aligned}
P(X)= & \frac{1}{S}\left(\sqrt{\frac{X}{B}}\right)^{m-1} \exp \left\{-\frac{1}{S}(X+B)\right\} \\
& \cdot I_{m-1}\left(\frac{2}{S} \sqrt{X B}\right) \quad(m=N / 2)
\end{aligned}
$$

(Bessel type distribution) .

On the other hand, by applying the method of moment, from Eq. (76), one can obtain the estimate of $m$ as follows;

$$
m=\frac{\Omega_{1}{ }^{2}-A^{4}}{\overline{R^{4}}-\Omega_{1}{ }^{2}}=\frac{m_{T}{ }^{2}-E_{0}{ }^{2}}{\sigma_{T}{ }^{2}} \quad\left(\sigma_{T}{ }^{2}=\left\langle\left(E-m_{T}\right)^{2}\right\rangle .\right.
$$

[2] For a special case with no regular component (i.e., $A=0$ ), we can easily have the following expressions:

$$
P(R)=\frac{2 m^{m}}{\Gamma(m) \Omega_{1}{ }^{m}} R^{2 m-1} \exp \left(-\frac{m R^{2}}{\Omega_{1}}\right),
$$

$$
\begin{aligned}
& F(\lambda)=\exp \left(-\frac{\Omega_{1}}{4 m} \lambda^{2}\right), \\
& \overline{R^{k}}=\sqrt{\left(\Omega_{1} / m\right)^{k}} \frac{\Gamma(m+k / 2)}{\Gamma(m)}
\end{aligned}
$$

respectively from Eqs. (71), (70) and (76). Furthermore, Eqs. (72) and (89) can be directly rewritten as follows:

$$
\begin{gathered}
P(E)=\frac{1}{\Gamma(m)}\left(\frac{m}{m_{T}}\right)^{m} E^{m-1} \exp \left(-\frac{m}{m_{T}} E\right), \\
P(X)=\frac{1}{\Gamma(m) s^{m}} X^{m-1} \exp \left(-\frac{X}{s}\right) \\
\quad \text { Gamma type distribution) }
\end{gathered}
$$

and especially for large values of $m$, the following expressions of probability density function are asymptotically obtained respectively as

$$
\begin{aligned}
& P(E) \equiv N\left(E ; m_{T}, \sigma_{T}^{2}\right), \\
& P(X) \equiv N\left(X ; \bar{X}, \sigma_{X}^{2}\right)
\end{aligned}
$$

with a variance $\sigma_{X}^{2}\left(\equiv\langle X-\bar{X})^{2}\right\rangle$. Corresponding to Eq. (90), a distribution parameter $m$ can be easily estimated based on the method of moment from Eq. (90) as

$$
m=\frac{\left(\overline{X)^{2}}\right.}{\sigma_{X}^{2}} .
$$

\section{EXPERIMENTAL CONSIDERATION}

In the preceding section, we have derived theoretically the expression of probability density function on the ultrasound intensity $E$ or its effective value $R$ in the presence of a specular signal component. In this section, we confirm the effectiveness of the above theory from the experimental viewpoint by applying it to the speckle pattern of clinical B-scan image actually for diagnostic purpose.

We have obtained experimental data by using a microdensitometer to scan film transparency images of ultrasound B-scans. The stepped gray scale included in the film image has been calibrated in terms of decibel, which was set on the basis of the most gray scale corresponding to the weakest ultrasound echo magnitude. Then, the speckle fluctuations in terms of photographic density was converted to fluctuation in the ultrasound echo magnitude.

In order to discuss superiority or inferiority among Rayleigh distribution, Rician distribution, Eq. (71), Eq. (91) and experimental frequency distribution, first, their distribution parameters have to be previously determined. A parameter $m(=N / 2)$ is directly obtained as $m=T W$ under the support of 
Shannon's sampling theorem from its definition by using a nominal bandwidth $W$ with a center frequency $3.5 \mathrm{MHz}$ of input ultrasound pulse used and a time constant of $T$ of square law detector. In this experiment, an estimate $m=2$ has been first obtained, and other parameters have been estimated by use of method of moment as follows:

A) for Rayleigh distribution (which letting $m=1$ in Eq. (91))

$$
\sigma_{R}^{2}=\left\langle R^{2}\right\rangle / 2,
$$

B) for Rician distribution (which letting $m=1$ in Eq. (71)

$$
\left.\begin{array}{c}
A=\sqrt{\left\langle R^{2}\right\rangle^{2}-\left\langle\left(R^{2}-\left\langle R^{2}\right\rangle\right)^{2}\right\rangle}, \\
\sigma_{R}^{2}=\left(\left\langle R^{2}\right\rangle-A^{2}\right) / 2,
\end{array}\right\}
$$

C) by using Eq. (71)

$$
\left.\begin{array}{c}
A=\sqrt{\left\langle R^{2}\right\rangle^{2}-\left\langle\left(R^{2}-\left\langle R^{2}\right\rangle\right)^{2}\right\rangle}, \\
\sigma_{R}{ }^{2}=\left(\left\langle R^{2}\right\rangle-A^{2}\right) / 2 m,
\end{array}\right\}
$$

D) by using Eq. (91)

$$
\sigma_{R}^{2}=\left\langle R^{2}\right\rangle / 2 m \text {. }
$$

Figure 4 shows a comparison between experimentally sampled values and theoretically evaluated curves for the cumulative probability distribution. It is obvious that Rayleigh distribution, Rician distribution and Eq. (91) (Gamma distribution) don't fit the experimental distribution at all. This reason seems

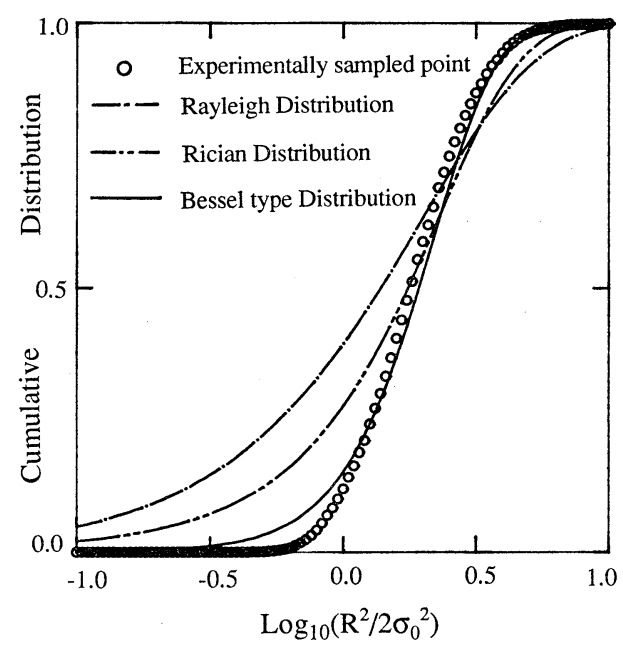

Fig. 4 A comparison between theoretically evaluated curves and experimentally sampled points of cumulative distribution for speckle pattern of B-mode image in vivo. to be as follows. Rayleigh distribution taking only sufficiently narrow frequency bandwidth into account and Eq. (91) taking frequency bandwidth $W$ and a time constant $T$ into account correspond originally to a special case of pure diffuse scattering, often to a fully developed speckle pattern. Rician distribution results from the combination of a strong specular component and weak random components, but the input signal is assumed to have a sufficiently narrow frequency bandwidth. On the other hand, with regard to the proposed distribution expression in Eq. (71) (Bessel type distribution), the fitness is quite better than the other three type distribution expressions. Obviously, this reason is that the effect of equipment and physical parameters, that is, a frequency bandwidth of input ultrasound pulse, a time constant of square law detector and the presence of specular scattering are explicitly reflected as distribution parameters.

\section{CONCLUSIONS}

In this paper, we have studied theoretically how the probability density function of the speckle image texture can be given in more actual case with presence of a specular component among the scattered waves. Here, the occurrence mechanism of speckle image texture has been treated as a probability problem of distance in an $N$-dimensional signal space with $N=2 T W$ (related to a time constant $T$ of square law detector and a frequency bandwidth $W$ of input ultrasound pulse) in direct connection with Shannon's sampling theorem. From the above point of view, the unified probability density function for the magnitude fluctuation of resultant vector (i.e., intensity) of incoherent ultrasound random waves and specular type coherent ultrasound waves, after passing a square law detector, has been theoretically derived. It has been found that the present theory completely agrees with the well-known Rayleigh and Rician distribution expressions as two special cases.

The main part of present research has been first focused on a new establishment of unified stochastic theory since it is at an early stage of study. So, there remain many kinds of problem on i) detecting some meaningful information for abnormal tissue buried under the speckle pattern, by finding some new types of stochastic image processing, ii) finding the quantitative evaluation method for the studying tissue characterization, iii) taking the effect of fre- 
quency dependent attenuation character into account, iv) finding the method to detect only the effective signal by using not only the above static type imaging processing but also the dynamic type digital filter, and so on.

\section{ACKNOWLEDGMENTS}

We would like to express our cordial thanks to K. Ito, K. Naito and K. Hayami for offering diagnostic ultrasound B-scan images and several helpful conversations.

\section{REFERENCES}

1) C. B. Burckhardt, "Speckle in ultrasound B mode scans," IEEE Trans. Sonics Ultrason. SU-25, 1-6 (1978).

2) J. G. Abbott and F. L. Thurstone, "Acoustic speckle; theory and experimental analysis," Ultrason. Imaging 1, 303-324 (1979).

3) D. L. Parker, "Analysis of B-scan speckle reduction by resolution limited filtering," Ultrason. Imaging 4, 108-125 (1982).

4) R.F. Wagner, S. W. Smith, J. M. Sandrik, and H. Lopez, "Statistics of speckle in ultrasound B-scans," IEEE Trans. Sonics Ultrason. SU-30, 156-163(1983).

5) S. W. Smith and R. F. Wagner, "Low contrast detectability and contrast/Detail analysis in medical ultrasound," IEEE Trans. Sonics Ultrason. SU-30, 164-173 (1983).

6) H. E. Melton Jr. and P. A. Magnin, "A-mode speckle reduction with compound frequency and compound bandwidth," Ultrason. Imaging 6, 159173 (1984).

7) R. F. Wagner, M. F. Insana, and S. W. Smith, "Fundamental correlation lengths of coherent speckle in medical ultrasonic images," IEEE Trans. Ultrason. Ferroelectr. Freq. Control UFFC-35, 3444 (1988).

8) L. Nock and G. E. Trahey, "Phase aberration correction in medical ultrasound using speckle brightness as a quality factor," J. Acoust. Soc. Am. 85, 1819-1833 (1989).

9) L. F. Joynt, "A stochastic approach to ultrasonic tissue characterization, Ph. D. thesis, Stanford Univ., also published as Tech. Rep. No. G557-4 (1979).

10) S. W. Smith and R. F. Wagner, "Ultrasound speckle size and lesion signal to noise ratio, verification of theory," Ultrason. Imaging 6, 174-180 (1984).

11) R. F. Wagner, M. F. Insana, and D. G. Brown "Unified approach to the detection and classification of speckle texture in diagnostic ultrasound," Opt. Eng. 25, 738-742 (1986).

12) M. F. Insana, R. F. Wagner, B. S. Garra, D. G. Brown, and T.H. Shawker, "Analysis of ultrasound image texture via generalized Rician statistics," Opt. Eng. 25, 743-748 (1986).
13) J. M. Thijssen and B. J. Oosterveld, "Speckle and texture in echography; artifact or information," Ultrason. Symp., 803-809 (1986).

14) S. Goldman, Information Theory (Prentice-Hall, New York, 1953).

15) M. Ohta, "A statistical treatment of the intensity fluctuation of random noise current," Mem. Coll. Sci. Univ. Kyoto Ser. XXX, 11 (1962).

16) K. Yoshida, T. Amemiya, K. Ito, T. Kato, K. Matsushima, and S. Furuya, Ed., Handbook of Applied Mathematics (Maruzen, Tokyo, 1967), p. 427 (iii) (in Japanese).

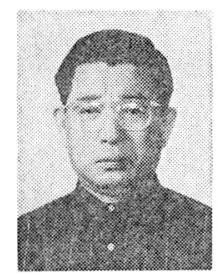

Mitsuo Ohta was born in 1928. He received a B. Sc. degree and a Dr. Sc. degree from the Kyoto Univ. in 1952 and 1961, respectively. After working as a research assistant and an associate professor in the Faculty of Eng. at Kobe and Fukui Univ's., and as a professor in the Faculty of Eng., at Fukui and Hiroshima Univ's., he has been a professor at the Faculty of Eng., Kinki Univ., since 1990. His research fields are the studies of micro-wave, dielectric, ultrasound, electro-magnetic-wave propagation, information processing, control theory, probabilistic engineering, sound and vibration control and digital filter. In 1966, he received a prize from ASJ for his paper. He is a member of ASJ, SICE, ISCIE, IEICE, IEEJ, and INCE Japan.

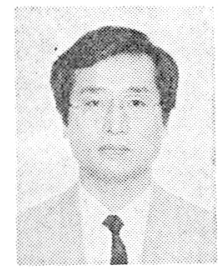

Shigeharu Miyata was born in 1958. He received a B.E., an M.E., and a Dr. Eng. degree from Hiroshima Univ. in 1981, 1983, and 1986, respectively. In 1986, he joined the Kinki University, Hiroshima, Japan, as a research assistant, and since 1990 he has been a lecturer in the Dept. of Mechanical Engineering. His research interest includes ultrasound, signal processing, probabilistic engineering, sound and vibration control and digital filter. $\mathrm{He}$ is a member of ASJ, SICE, ISCIE, IEICE and JSME.

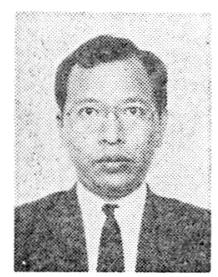

Noboru Nakasako was born in 1960. He received a B.E., an M.E., and a Dr. Eng. degree from Hiroshima Univ. in 1982, 1984, and 1990, respectively. In 1986, he joined the Hiroshima Institute of Technology, Hiroshima, Japan, as a research assistant, and since 1990 he has been an assistant professor in the Dept. of Electrical Engineering. His research interest includes ultrasound, electro-magnetic-wave propagation, information processing, control theory, probabilistic engineering, sound and vibration control and digital filter. He is a member of ASJ, SICE, ISCIE, IEICE, JSAA and INCE Japan. 